Opuscula Mathematica • Vol. $30 \bullet$ No. $4 \bullet 2010$

http://dx.doi.org/10.7494/OpMath.2010.30.4.411

\title{
EXISTENCE AND ATTRACTIVITY RESULTS FOR NONLINEAR FIRST ORDER RANDOM DIFFERENTIAL EQUATIONS
}

\author{
Bapurao C. Dhage, Sotiris K. Ntouyas
}

\begin{abstract}
In this paper, the existence and attractivity results are proved for nonlinear first order ordinary random differential equations. Two examples are provided to demonstrate the realization of the abstract developed theory.
\end{abstract}

Keywords: random differential equations, locally attractive, globally attractive, local asymptotic attractive, fixed point theorem.

Mathematics Subject Classification: 47H40, 60H25.

\section{INTRODUCTION}

Let $\mathbb{R}$ denote the real line and $\mathbb{R}_{+}$, the set of nonnegative real numbers, that is, $\mathbb{R}_{+}=[0, \infty) \subset \mathbb{R}$. Let $C\left(\mathbb{R}_{+}, \mathbb{R}\right)$ denote the class of real-valued functions defined and continuous on $\mathbb{R}_{+}$. Given a measurable space $(\Omega, \mathcal{A})$ and a measurable function $x: \Omega \rightarrow C\left(\mathbb{R}_{+}, \mathbb{R}\right)$, we consider the initial value problem of nonlinear first order ordinary random differential equations (in short $\mathrm{RDE}$ )

$$
\left\{\begin{aligned}
x^{\prime}(t, \omega)+k(t, \omega) x(t, \omega) & =f(t, x(t, \omega), \omega) \text { a.e. } t \in \mathbb{R}_{+}, \\
x(0, \omega) & =q(\omega)
\end{aligned}\right.
$$

for all $\omega \in \Omega$, where $k: \mathbb{R}_{+} \times \Omega \rightarrow \mathbb{R}_{+}, q: \Omega \rightarrow \mathbb{R}$ and $f: \mathbb{R}_{+} \times \mathbb{R} \times \Omega \rightarrow \mathbb{R}$

By a random solution of the RDE (1.1) we mean a measurable function $x$ : $\Omega \rightarrow A C\left(\mathbb{R}_{+}, \mathbb{R}\right)$ that satisfies the equations in $(1.1)$, where $A C\left(\mathbb{R}_{+}, \mathbb{R}\right)$ is the space of absolutely continuous real-valued functions defined on $\mathbb{R}_{+}$.

The initial value problems of ordinary differential equations have been studied in the literature on bounded as well as unbounded internals of the real line for different aspects of the solution. See for example, Burton and Furumochi [2], Dhage [5, 6], Hu and Yan [7] and the references therein. Similarly, the initial value problem of random 
differential equations have also been discussed in the literature for existence theorems on bounded intervals, however, the study of such random equations has not been made on unbounded intervals of the real line for any aspects of the random solutions. Some results along this lines appear in Itoh [8], Bharucha-Reid [1] and Dhage [4].

Therefore, nonlinear random differential equations on unbounded intervals need to pay attention to the existence as well as the different characterizations of the random solutions. The present paper proposes to discuss the existence and attractivity results for random differential equations (1.1) on the right half $\mathbb{R}_{+}$of the real line $\mathbb{R}$. The classical fixed point theory, in particular, random version of Schauder's fixed point theorem will be employed to prove the main result of this paper. Our results generalize the stability results of Burton and Furumochi [2] in some sense and we claim that the results of this paper are new to the literature on random differential equations.

\section{AUXILIARY RESULTS}

Let $E$ denote a Banach space with the norm $\|\cdot\|$ and let $Q: E \rightarrow E$ be an operator (in general nonlinear). Then $Q$ is called:

(i) compact if $Q(E)$ is a relatively compact subset of $E$;

(ii) totally bounded if $Q(B)$ a is totally bounded subset of $E$ for any bounded subset $B$ of $E$;

(iii) completely continuous if it is continuous and totally bounded on $E$.

Note that every compact operator is totally bounded, but the converse may not be true.

We further assume that the Banach space $E$ is separable, i.e., $E$ has a countable dense subset and let $\beta_{E}$ be the $\sigma$-algebra of the Borel subsets of $E$. We say a mapping $x: \Omega \rightarrow E$ is measurable if for any $B \in \beta_{E}$,

$$
x^{-1}(B)=\{\omega \in \Omega \mid x(\omega) \in B\} \in \mathcal{A} .
$$

Note that a continuous map $f$ from a Banach space $E$ into itself is measurable, but the converse may not be true. Let $Q: \Omega \times E \rightarrow E$ be a mapping. Then $Q$ is called a random operator if $Q(\omega, x)$ is measurable in $\omega$ for all $x \in E$ and it expressed as $Q(\omega) x=Q(\omega, x)$. In this case we also say that $Q(\omega)$ is a random operator on $E$. A random operator $Q(\omega)$ on $E$ is called continuous (resp. compact, totally bounded and completely continuous) if $Q(\omega, x)$ is continuous (resp. compact, totally bounded and completely continuous) in $x$ for all $\omega \in \Omega$. The details of completely continuous random operators on Banach spaces appear in Itoh [8]. We employ the following random fixed point theorem as a tool while formulating and proving our main existence results for the $\operatorname{RDE}(1.1)$ on $\mathbb{R}_{+}$.

Theorem 2.1 (Itoh [8]). Let $X$ be a non-empty, closed convex bounded subset of the separable Banach space $E$ and let $Q: \Omega \times X \rightarrow X$ be a compact and continuous random operator. Then the random equation $Q(\omega) x=x$ has a random solution, i.e., there is a measurable function $\xi: \Omega \rightarrow X$ such that $Q(\omega) \xi(\omega)=\xi(\omega)$ for all $\omega \in \Omega$. 
The following theorem is often used in the study of nonlinear discontinuous random differential equations. We also need this result in the subsequent part of this paper.

Theorem 2.2 (Carathéodory). Let $Q: \Omega \times E \rightarrow E$ be a mapping such that $Q(\cdot, x)$ is measurable for all $x \in E$ and $Q(\omega, \cdot)$ is continuous for all $\omega \in \Omega$. Then the map $(\omega, x) \mapsto Q(\omega, x)$ is jointly measurable.

\section{CHARACTERIZATIONS OF RANDOM SOLUTIONS}

We seek the random solutions of $\operatorname{RDE}(1.1)$ in the Banach space $B C\left(\mathbb{R}_{+}, \mathbb{R}\right)$ of real-valued functions defined, continuous and bounded on $\mathbb{R}_{+}$. We equip the space $B C\left(\mathbb{R}_{+}, \mathbb{R}\right)$ with the supremum norm $\|\cdot\|$ defined by

$$
\|x\|=\sup _{t \in \mathbb{R}_{+}}|x(t)| .
$$

It is known that the Banach space $B C\left(\mathbb{R}_{+}, \mathbb{R}\right)$ is separable. By $L^{1}\left(\mathbb{R}_{+}, \mathbb{R}\right)$ we denote the space of Lebesgue measurable real-valued functions defined on $\mathbb{R}_{+}$. By $\|\cdot\|_{L^{1}}$ we denote the usual norm in $L^{1}\left(\mathbb{R}_{+}, \mathbb{R}\right)$ defined by

$$
\|x\|_{L^{1}}=\int_{0}^{\infty}|x(t)| d t .
$$

In order to introduce the further concepts used in this paper, let us denote $E=$ $B C\left(\mathbb{R}_{+}, \mathbb{R}\right)$ and $S$ be a non-empty subset of $E$. Let $Q: \Omega \times E \rightarrow E$ be a mapping and consider the following random equation

$$
Q(\omega) x(t)=x(t, \omega)
$$

for $t \in \mathbb{R}_{+}$and $\omega \in \Omega$. A measurable function $x: \Omega \rightarrow E$ is called a random solution of the random equation (3.1) if it satisfies (3.1) on $\mathbb{R}_{+}$. Below we give different characterizations of the random solutions for the random equation (3.1) on $\mathbb{R}_{+}$.

Definition 3.1. We say that random solutions of the random equation (3.1) are locally attractive on $\mathbb{R}_{+}$if there exists a closed ball $\overline{\mathcal{B}}_{r}\left(x_{0}\right)$ in the space $B C\left(\mathbb{R}_{+}, \mathbb{R}\right)$ for some $x_{0} \in B C\left(\mathbb{R}_{+}, \mathbb{R}\right)$ and for some real number $r>0$, such that for arbitrary random solutions $x=x(t, \omega)$ and $y=y(t, \omega)$ of the random equation (3.1) belonging to $\overline{\mathcal{B}}_{r}\left(x_{0}\right) \cap S$ we have that

$$
\lim _{t \rightarrow \infty}(x(t, \omega)-y(t, \omega))=0
$$

for all $\omega \in \Omega$. In this case when the limit (3.2) is uniform with respect to the set $\overline{\mathcal{B}}_{r}\left(x_{0}\right) \cap S$, that is, when for each $\varepsilon>0$ there exists a $T>0$ such that for all $t \geq T$,

$$
|x(t, \omega)-y(t, \omega)| \leq \varepsilon
$$

for all $\omega \in \Omega$ and for all $x, y \in \overline{\mathcal{B}}_{r}\left(x_{0}\right) \cap S$ being the random solutions of (3.1), we will say that the random solutions are uniformly locally attractive on $\mathbb{R}_{+}$. 
Definition 3.2. We say that random solutions of the random equation (3.1) are globally attractive on $\mathbb{R}_{+}$if for arbitrary random solutions $x=x(t, \omega)$ and $y=y(t, \omega)$ of the random equation (3.1) belonging to $S$ the condition (3.2) is satisfied. In the case when (3.2) is satisfied uniformly with respect to the set $S$ in $E$, that is, for $\varepsilon>0$ there exists a $T>0$ such that $t \geq T$, the inequality (3.3) holds for all $x, y \in S$ being the random solutions for the random equation (3.1), we will say that the random solutions of the random equation (3.1) are uniformly globally attractive on $\mathbb{R}_{+}$.

Definition 3.3. Let $c \in \mathbb{R}$ be fixed. A line $y(t, \omega)=c$ for all $t \in \mathbb{R}_{+}$and $\omega \in \Omega$, is called an attractor for the random solution $x: \Omega \rightarrow E$ to the random equation (3.1) if $\lim _{t \rightarrow \infty}[x(t, \omega)-c]=0$ for all $\omega \in \Omega$. In this case, the random solution $x$ to the random equation (3.1) is said to be asymptotic to the line $y=c$ and the line is an asymptote for the random solution $x$ on $\mathbb{R}_{+}$.

Definition 3.4. The random solutions for the random equation (3.1) are said to be locally asymptotically attractive if there exists a closed ball $\overline{\mathcal{B}}_{r}\left(x_{0}\right)$ in $E$ for some $x_{0} \in E$ and for some real number $r>0$, such that for any two random solutions $x=x(t, \omega)$ and $y=y(t, \omega)$ to the random equation (3.1) belonging to $\overline{\mathcal{B}}_{r}\left(x_{0}\right) \cap S$, there is a line which is a common attractor to them on $\mathbb{R}_{+}$. When $x$ and $y$ are uniformly locally attractive and there is a line as a common attractor, we will say that the random solutions of the random equation (3.1) are uniformly locally attractive on $\mathbb{R}_{+}$.

Definition 3.5. The random solutions for the random equation (3.1) are said to be globally asymptotically attractive if for any two globally attractive solutions $x$ and $y$ of (3.1) there is a line which is a common attractor to them on $\mathbb{R}_{+}$. Furthermore, if the random solutions for the random equation (3.1) are uniformly globally attractive, then they are called uniformly globally asymptotically attractive on $\mathbb{R}_{+}$.

We note that the global attractivity and global asymptotic attractivity implies respectively the local attractivity and local asymptotic attractivity of the random solutions. The same is also true for uniformly globally attractivity and uniformly globally asymptotic attractivity of the random solutions for the random equation (3.1). However, the reverse implication may not hold.

\section{ATTRACTIVITY RESULTS}

We need the following definition in the sequel.

Definition 4.1. A function $f: \mathbb{R}_{+} \times \mathbb{R} \times \Omega \rightarrow \mathbb{R}$ is called random Carathéodory if:

(i) the map $\omega \mapsto f(t, x, \omega)$ is measurable for all $t \in \mathbb{R}_{+}$and $x \in \mathbb{R}$ and

(ii) the map $(t, x) \mapsto f(t, x, \omega)$ is jointly continuous for all $\omega \in \Omega$.

Furthermore, a random Carathéodory function $f: \mathbb{R}_{+} \times \mathbb{R} \times \Omega \rightarrow \mathbb{R}$ is called random $L^{1}$-Carathéodory, if there exists a function $h \in L^{1}\left(\mathbb{R}_{+}, \mathbb{R}\right)$ such that

$$
|f(t, x, \omega)| \leq h(t) \text { a.e. } t \in \mathbb{R}_{+}
$$

for all $\omega \in \Omega$ and $x \in \mathbb{R}$. The function $h$ is the growth function of $f$ on $\mathbb{R}_{+} \times \mathbb{R} \times \Omega$. 
We consider the following set of hypotheses in what follows.

$\left(\mathrm{H}_{0}\right)$ The function $k: \mathbb{R}_{+} \times \mathbb{R} \rightarrow \mathbb{R}$ is measurable and bounded. Moreover, for each $\omega \in \Omega$

$$
\lim _{t \rightarrow \infty} K(t, \omega)=\infty
$$

where $K(t, \omega):=\int_{0}^{t} k(s, \omega) d s$.

$\left(\mathrm{H}_{1}\right)$ The function $q: \Omega \rightarrow \mathbb{R}$ is measurable and bounded. Moreover,

$$
\text { ess } \sup _{\omega \in \Omega}|q(\omega)|=c_{1}
$$

for some real number $c_{1}>0$.

$\left(\mathrm{H}_{2}\right)$ The function $f$ is random $L^{1}$-Carathéodory with growth function $h$ on $\mathbb{R}_{+}$. Moreover,

$$
\lim _{t \rightarrow \infty} e^{-K(t, \omega)} \int_{0}^{t} e^{K(s, \omega)} h(s) d s=0
$$

for all $\omega \in \Omega$.

Remark 4.2. If the hypothesis $\left(\mathrm{H}_{2}\right)$ holds, then the function $w: \mathbb{R}_{+} \times \Omega \rightarrow \mathbb{R}_{+}$ defined by $w(t, \omega)=e^{-K(t, \omega)} \int_{0}^{t} e^{K(s, \omega)} h(s) d s$ is continuous and the number

$$
W=\sup _{t \geq 0} w(t, \omega)=\sup _{t \geq 0} e^{-K(t, \omega)} \int_{0}^{t} e^{K(s, \omega)} h(s) d s
$$

exists for all $\omega \in \Omega$. Hypothesis $\left(\mathrm{H}_{2}\right)$ has been considered in a number of papers in the literature. See for example, Dhage [6], Burton and Furumochi [2] and the references therein.

Our main result is the following:

Theorem 4.3. Assume that the hypotheses $\left(\mathrm{H}_{0}\right)$ through $\left(\mathrm{H}_{2}\right)$ hold. Then the RDE (1.1) admits a random solution. Moreover, random solutions are uniformly globally asymptotically attractive to the zero random solution on $\mathbb{R}_{+}$.

Proof. Now RDE (1.1) is equivalent to the random equation

$$
x(t, \omega)=q(\omega) e^{-K(t, \omega)}+e^{-K(t, \omega)} \int_{0}^{t} e^{K(s, \omega)} f(s, x(s, \omega), \omega) d s
$$

for all $t \in \mathbb{R}_{+}$and $\omega \in \Omega$. 
Set $E=B C\left(\mathbb{R}_{+}, \mathbb{R}\right)$. For a given function $x: \Omega \rightarrow E$, define a mapping $Q$ on $\Omega \times E$ by

$$
Q(\omega) x(t, \omega)=q(\omega) e^{-K(t, \omega)}+e^{-K(t, \omega)} \int_{0}^{t} e^{K(s, \omega)} f(s, x(s, \omega), \omega) d s
$$

for all $t \in \mathbb{R}_{+}$and $\omega \in \Omega$. For the sake of convenience, we write $Q(\omega) x(t, \omega)=$ $Q(\omega) x(t)$ omitting the double appearance of $\omega$ merge it into $Q(\omega)$.

Clearly, $Q$ defines a mapping $Q: \Omega \times E \rightarrow E$. To see this, let $x \in E$ be arbitrary. Then for each $\omega \in \Omega$, the continuity of map $t \mapsto Q(\omega) x(t)$ follows from the fact that exponential $e^{-K(t, \omega)}$ and the indefinite integral $\int_{0}^{t} f(s, x(s, \omega), \omega) d s$ are continuous functions of $t$ on $\mathbb{R}_{+}$. Next, we show that the function $Q(\omega) x: \mathbb{R}_{+} \rightarrow \mathbb{R}$ is bounded for each $\omega \in \Omega$. Now by hypotheses $\left(\mathrm{H}_{1}\right)$ and $\left(\mathrm{H}_{2}\right)$,

$$
\begin{aligned}
|Q(\omega) x(t)| & \leq|q(\omega)| e^{-K(t, \omega)}+e^{-K(t, \omega)} \int_{0}^{t} e^{K(s, \omega)}|f(s, x(s, \omega), \omega)| d s \leq \\
& \leq c_{1} e^{-K(t, \omega)}+e^{-K(t, \omega)} \int_{0}^{t} e^{K(s, \omega)} h(s) d s \leq c_{1}+W
\end{aligned}
$$

for all $\omega \in \Omega$. As a result, $Q: \Omega \times E \rightarrow E$.

Define a closed ball $\overline{\mathcal{B}}_{r}(0)$ in the Banach space $E$ centered at origin of radius $r=c_{1}+W$. From (4.3),

$$
\|Q(\omega) x\| \leq c_{1}+W
$$

for all $\omega \in \Omega$ and $x \in E$. Hence $Q: \Omega \times E \rightarrow \overline{\mathcal{B}}_{r}(0)$, and in particular, $Q$ defines a map $Q: \Omega \times \overline{\mathcal{B}}_{r}(0) \rightarrow \overline{\mathcal{B}}_{r}(0)$. Now we show that $Q$ satisfies all the conditions of Theorem 2.1 with $X=\overline{\mathcal{B}}_{r}(0)$.

Firstly, we show that $Q$ is a random operator on $\Omega \times \overline{\mathcal{B}}_{r}(0)$ into $\overline{\mathcal{B}}_{r}(0)$. By hypothesis $\left(\mathrm{H}_{2}\right)$, the map $\omega \mapsto f(t, x, \omega)$ is measurable by the Carathéodory theorem. Since a continuous function is measurable, the map $t \mapsto e^{K(t, \omega)}$ is measurable and so the product $e^{K(t, \omega)} f(t, x(t, \omega), \omega)$ is measurable in $\omega$ for all $t \in \mathbb{R}_{+}$and $x \in \mathbb{R}$. Since the integral is a limit of the finite sum of measurable functions, we have that the function $\omega \mapsto \int_{0}^{t} e^{K(s, \omega)} f(s, x(s, \omega), \omega) d s$ is measurable. Similarly, the map

$$
\omega \mapsto q(\omega) e^{-K(t, \omega)}+e^{-K(t, \omega)} \int_{0}^{t} e^{K(s, \omega)} f(s, x(s, \omega), \omega) d s
$$

is measurable for all $t \in \mathbb{R}_{+}$. Consequently, the map $\omega \mapsto Q(\omega) x$ is measurable for all $x \in E$ and that $Q$ is a random operator on $\Omega \times \overline{\mathcal{B}}_{r}(0)$.

Secondly, we show that the random operator $Q(\omega)$ is continuous on $\overline{\mathcal{B}}_{r}(0)$. By hypothesis $\left(\mathrm{H}_{2}\right), \lim _{t \rightarrow \infty} w(t, \omega)=\lim _{t \rightarrow \infty} e^{-K(t, \omega)} \int_{0}^{t} e^{K(s, \omega)} h(s) d s=0$, there is a real number $T>0$ such that $w(t)<\frac{\varepsilon}{4}$ for all $t \geq T$. 
We show the continuity of the random operator $Q(\omega)$ in the following two cases: Case $I$. Let $t \in[0, T]$ and let $\left\{x_{n}\right\}$ be a sequence of points in $\overline{\mathcal{B}}_{r}(0)$ such that $x_{n} \rightarrow x$ as $n \rightarrow \infty$. Then, by the dominated convergence theorem,

$$
\begin{aligned}
\lim _{n \rightarrow \infty} Q(\omega) x_{n}(t) & =\lim _{n \rightarrow \infty}\left(q(\omega) e^{-K(t, \omega)}+e^{-K(t, \omega)} \int_{0}^{t} e^{K(s, \omega)} f\left(s, x_{n}(s, \omega), \omega\right) d s\right)= \\
& =q(\omega) e^{-K(t, \omega)}+\lim _{n \rightarrow \infty}\left(e^{-K(t, \omega)} \int_{0}^{t} e^{K(s, \omega)} f\left(s, x_{n}(s, \omega), \omega\right) d s\right)= \\
& =q(\omega) e^{-K(t, \omega)}+e^{-K(t, \omega)} \int_{n \rightarrow \infty}^{t} \lim _{0}^{t}\left[e^{K(s, \omega)} f\left(s, x_{n}(s, \omega), \omega\right)\right] d s= \\
& =q(\omega) e^{-K(t, \omega)}+e^{-K(t, \omega)} \int_{0}^{t} e^{K(s, \omega)} f(s, x(s, \omega), \omega) d s= \\
& =Q(\omega) x(t)
\end{aligned}
$$

for all $t \in[0, T]$ and $\omega \in \Omega$.

Case II. Suppose that $t \geq T$. Then we have:

$$
\begin{aligned}
& \left|Q(\omega) x_{n}(t)-Q(\omega) x(t)\right|= \\
& =\left|e^{-K(t, \omega)} \int_{0}^{t} e^{K(s, \omega)} f\left(s, x_{n}(s, \omega), \omega\right) d s-e^{-K(t, \omega)} \int_{0}^{t} e^{K(s, \omega)} f(s, x(s, \omega), \omega) d s\right| \leq \\
& \leq\left|e^{-K(t, \omega)} \int_{0}^{t} e^{K(s, \omega)} f\left(s, x_{n}(s, \omega), \omega\right) d s\right|+\left|e^{-K(t, \omega)} \int_{0}^{t} e^{K(s, \omega)} f(s, x(s, \omega), \omega) d s\right| \leq \\
& \leq 2 w(t)<\varepsilon
\end{aligned}
$$

for all $t \geq T$ and $\omega \in \Omega$. Since $\varepsilon$ is arbitrary, one has $\lim _{n \rightarrow \infty} Q(\omega) x_{n}(t)=Q(\omega) x(t)$ for all $t \geq T$ and $\omega \in \Omega$. Now combining the Case I with Case II, we conclude that $Q(\omega)$ is a pointwise continuous random operator on $\overline{\mathcal{B}}_{r}(0)$ into itself. Further, it is shown below that the family of functions $\left\{Q(\omega) x_{n}\right\}$ is an equicontinuous set in $E$ for a fixed $\omega \in \Omega$. Hence, the above convergence is uniform on $\mathbb{R}_{+}$and consequently, $Q(\omega)$ is a continuous random operator on $\overline{\mathcal{B}}_{r}(0)$ into itself.

Next, we show that $Q(\omega)$ is a compact random operator on $\overline{\mathcal{B}}_{r}(0)$. Let $\omega \in \Omega$ be fixed and consider a sequence $\left\{Q(\omega) x_{n}\right\}$ of points in $\overline{\mathcal{B}}_{r}(0)$. To finish, it is enough to show that the sequence $\left\{Q(\omega) x_{n}\right\}$ has a Cauchy subsequence for each $\omega \in \Omega$. Clearly, $\left\{Q(\omega) x_{n}\right\}$ is a uniformly bounded subset of $\overline{\mathcal{B}}_{r}(0)$. 
We show that it is an equi-continuous sequence of functions on $\mathbb{R}_{+}$.

Let $\varepsilon>0$ be given. Since $\lim _{t \rightarrow \infty} w(t, \omega)=0$, there exists a real number $T>0$ such that $w(t)<\frac{\varepsilon}{4}$ for $t \geq T$. We consider the following three cases:

Case $I$. Let $t_{1}, t_{2} \in[0, T]$. Then we have

$$
\begin{aligned}
& \left|Q(\omega) x_{n}\left(t_{1}\right)-Q(\omega) x_{n}\left(t_{2}\right)\right| \leq \\
& \leq \mid q(\omega) e^{-K\left(t_{1}, \omega\right)}+e^{-K\left(t_{1}, \omega\right)} \int_{0}^{t_{1}} e^{K(s, \omega)} f\left(s, x_{n}(s, \omega), \omega\right) d s- \\
& -q(\omega) e^{-K\left(t_{2}, \omega\right)}-e^{-K\left(t_{2}, \omega\right)} \int_{0}^{t_{2}} e^{K(s, \omega)} f\left(s, x_{n}(s, \omega), \omega\right) d s \mid \leq \\
& \leq|q(\omega)|\left|e^{-K\left(t_{1}, \omega\right)}-e^{-K\left(t_{2}, \omega\right)}\right|+ \\
& +\left|e^{-K\left(t_{1}, \omega\right)} \int_{0}^{t_{1}} e^{K(s, \omega)} f\left(s, x_{n}(s, \omega), \omega\right) d s-e^{-K\left(t_{2}, \omega\right)} \int_{0}^{t_{1}} e^{K(s, \omega)} f\left(s, x_{n}(s, \omega), \omega\right) d s\right|+ \\
& +\left|e^{-K\left(t_{2}, \omega\right)} \int_{0}^{t_{1}} e^{K(s, \omega)} f\left(s, x_{n}(s, \omega), \omega\right) d s-e^{-K\left(t_{2}, \omega\right)} \int_{0}^{t_{2}} e^{K(s, \omega)} f(s, x(s, \omega), \omega) d s\right| \leq \\
& \leq|q(\omega)|\left|e^{-K\left(t_{1}, \omega\right)}-e^{-K\left(t_{2}, \omega\right)}\right|+ \\
& +\left|e^{-K\left(t_{1}, \omega\right)}-e^{-K\left(t_{2}, \omega\right)}\right|\left|\int_{0}^{t_{1}} e^{K(s, \omega)} f\left(s, x_{n}(s, \omega), \omega\right) d s\right|+ \\
& +e^{-K\left(t_{2}, \omega\right)}\left|\int_{0}^{t_{1}} e^{K(s, \omega)} f\left(s, x_{n}(s, \omega), \omega\right) d s-\int_{0}^{t_{2}} e^{K(s, \omega)} f\left(s, x_{n}(s, \omega), \omega\right) d s\right| \leq \\
& \leq\left[|q(\omega)|+\int_{0}^{T} e^{K(s, \omega)} h(s) d s\right]\left|e^{-K\left(t_{1}, \omega\right)}-e^{-K\left(t_{2}, \omega\right)}\right|+ \\
& +e^{-K\left(t_{2}, \omega\right)}\left|\int_{t_{2}}^{t_{1}} e^{K(s, \omega)}\right| f\left(s, x_{n}(s, \omega), \omega\right)|d s| \leq \\
& \leq\left[c_{1}+e^{k_{1}}\|h\|_{L^{1}}\right]\left|e^{-K\left(t_{1}, \omega\right)}-e^{-K\left(t_{2}, \omega\right)}\right|+\mid p\left(t_{1}\right)-p\left(t_{2}\right)
\end{aligned}
$$

for all $n \in \mathbb{N}$, where $k_{1}$ is the bound for $k(t, \omega)$ and $p(t)=\int_{0}^{t} e^{K(s, \omega)} h(s) d s$. Since the functions $e^{-K(t, \omega)}$ and $p(t)$ are continuous on $[0, T]$, they are uniformly continuous 
there. Hence,

$$
\left|Q(\omega) x_{n}\left(t_{1}\right)-Q(\omega) x_{n}\left(t_{2}\right)\right| \rightarrow 0 \quad \text { as } \quad t_{1} \rightarrow t_{2}
$$

uniformly for all $t_{1}, t_{2} \in[0, T]$ and for all $n \in \mathbb{N}$.

Case II. If $t_{1}, t_{2} \in[T, \infty)$, then we have

$$
\begin{aligned}
& \left|Q(\omega) x_{n}\left(t_{1}\right)-Q(\omega) x_{n}\left(t_{2}\right)\right| \leq \\
& \leq\left|e^{-K\left(t_{1}, \omega\right)} \int_{0}^{t_{1}} e^{K(s, \omega)} f\left(s, x_{n}(s, \omega), \omega\right) d s-e^{-K\left(t_{2}, \omega\right)} \int_{0}^{t_{2}} e^{K(s, \omega)} f\left(s, x_{n}(s, \omega), \omega\right) d s\right| \leq \\
& \leq e^{-K\left(t_{1}, \omega\right)} \int_{0}^{t_{1}} e^{K(s, \omega)}\left|f\left(s, x_{n}(s, \omega), \omega\right)\right| d s+e^{-K\left(t_{2}, \omega\right)} \int_{0}^{t_{2}} e^{K(s, \omega)}\left|f\left(s, x_{n}(s, \omega), \omega\right)\right| d s \leq \\
& \leq w\left(t_{1}\right)+w\left(t_{2}\right) \leq \frac{\varepsilon}{4}+\frac{\varepsilon}{4}<\varepsilon .
\end{aligned}
$$

Since $\varepsilon$ is arbitrary, one has

$$
\left|Q(\omega) x_{n}\left(t_{1}\right)-Q(\omega) x_{n}\left(t_{2}\right)\right| \rightarrow 0 \quad \text { as } \quad t_{1} \rightarrow t_{2}
$$

uniformly for all $n \in \mathbb{N}$.

Case III. If $t_{1}<T<t_{2}$, then

$$
\begin{aligned}
\left|Q(\omega) x_{n}\left(t_{1}\right)-Q(\omega) x_{n}\left(t_{2}\right)\right| \leq & \left|Q(\omega) x_{n}\left(t_{1}\right)-Q(\omega) x_{n}(T)\right|+ \\
& +\left|Q(\omega) x_{n}(T)-Q(\omega) x_{n}\left(t_{2}\right)\right| .
\end{aligned}
$$

As $t_{1} \rightarrow t_{2}, t_{1} \rightarrow T$ and $t_{2} \rightarrow T$, and so

$$
\left|Q(\omega) x_{n}\left(t_{1}\right)-Q(\omega) x_{n}(T)\right| \rightarrow 0
$$

and

$$
\left|Q(\omega) x_{n}\left(t_{2}\right)-Q(\omega) x_{n}(T)\right| \rightarrow 0
$$

as $t_{1} \rightarrow t_{2}$ uniformly for all $n \in \mathbb{N}$. Hence,

$$
\left|Q(\omega) x_{n}\left(t_{1}\right)-Q(\omega) x_{n}\left(t_{2}\right)\right| \rightarrow 0 \quad \text { as } \quad t_{1} \rightarrow t_{2}
$$

uniformly for all $t_{1}<T, t_{2}>T$ and for all $n \in \mathbb{N}$. Thus, in all three cases

$$
\left|Q(\omega) x_{n}\left(t_{1}\right)-Q(\omega) x_{n}\left(t_{2}\right)\right| \rightarrow 0 \quad \text { as } \quad t_{1} \rightarrow t_{2}
$$

uniformly for all $t_{1}, t_{2} \in \mathbb{R}_{+}$and for all $n \in \mathbb{N}$.

This shows that $\left\{Q(\omega) x_{n}\right\}$ is a equicontinuous sequence in $X$. Now an application of Arzelá-Ascoli theorem yields that $\left\{Q(\omega) x_{n}\right\}$ has a uniformly convergent subsequence on the compact subset $[0, T]$ of $\mathbb{R}$. Without loss of generality, call the subsequence to be the sequence itself. We show that $\left\{Q(\omega) x_{n}\right\}$ is Cauchy in $X$. Now 
$\left|Q(\omega) x_{n}(t)-Q(\omega) x(t)\right| \rightarrow 0$ as $n \rightarrow \infty$ for all $t \in[0, T]$. Then for given $\varepsilon>0$ there exits an $n_{0} \in \mathbb{N}$ such that

$$
\sup _{0 \leq p \leq T} e^{k_{1} p} \int_{0}^{p} e^{K(s, \omega)}\left|f\left(s, x_{m}(s, \omega), \omega\right)-f\left(s, x_{n}(s, \omega), \omega\right)\right| d s<\frac{\varepsilon}{2}
$$

for all $m, n \geq n_{0}$. Therefore, if $m, n \geq n_{0}$, then we have

$$
\begin{aligned}
& \left\|Q(\omega) x_{m}-Q(\omega) x_{n}\right\|= \\
& =\sup _{0 \leq t<\infty}\left|e^{-K(t, \omega)} \int_{0}^{t} e^{K(s, \omega)}\right| f\left(s, x_{m}(s, \omega), \omega\right)-f\left(s, x_{n}(s, \omega), \omega\right)|d s| \leq \\
& \leq \sup _{0 \leq p \leq T}\left|e^{-K(p, \omega)} \int_{0}^{p} e^{K(s, \omega)}\right| f\left(s, x_{m}(s, \omega), \omega\right)-f\left(s, x_{n}(s, \omega), \omega\right)|d s|+ \\
& \quad+\sup _{p \geq T} e^{-K(p, \omega)} \int_{0}^{p} e^{K(s, \omega)}\left[\left|f\left(s, x_{m}(s, \omega), \omega\right)\right|+\left|f\left(s, x_{n}(s, \omega), \omega\right)\right|\right] d s<\varepsilon .
\end{aligned}
$$

This shows that $\left\{Q(\omega) x_{n}\right\} \subset Q(\omega)\left(\overline{\mathcal{B}}_{r}(0)\right) \subset \overline{\mathcal{B}}_{r}(0)$ is Cauchy. Since $X$ is complete, $\left\{Q(\omega) x_{n}\right\}$ converges to a point in $X$. As $Q(\omega)\left(\overline{\mathcal{B}}_{r}(0)\right)$ is closed, the sequence $\left\{Q(\omega) x_{n}\right\}$ converges to a point in $Q(\omega)\left(\overline{\mathcal{B}}_{r}(0)\right)$. Hence $Q(\omega)\left(\overline{\mathcal{B}}_{r}(0)\right)$ is relatively compact for each $\omega \in \Omega$ and consequently $Q$ is a continuous and compact random operator on $\Omega \times \overline{\mathcal{B}}_{r}(0)$ into $\overline{\mathcal{B}}_{r}(0)$. Now an application of Theorem 2.1 to the operator $Q(\omega)$ on $\overline{\mathcal{B}}_{r}(0)$ yields that $Q$ has a fixed point in $\overline{\mathcal{B}}_{r}(0)$ which further implies that the RDE (1.1) has a random solution on $\mathbb{R}_{+}$.

Next, we show that the solutions are uniformly attractive on $\mathbb{R}_{+}$. Let $x, y: \Omega \rightarrow$ $\overline{\mathcal{B}}_{r}(0)$ be any two random solutions to the $\operatorname{RDE}(1.1)$ on $\mathbb{R}_{+}$. Then, for each $\omega \in \Omega$,

$$
\begin{aligned}
& |x(t, \omega)-y(t, \omega)| \leq \\
& \leq\left|e^{-K(t, \omega)} \int_{0}^{t} e^{K(s, \omega)} f(s, x(s, \omega), \omega) d s-e^{-K(t, \omega)} \int_{0}^{t} e^{K(s, \omega)} f(s, y(s, \omega), \omega) d s\right| \leq \\
& \leq e^{-K(t, \omega)} \int_{0}^{t} e^{K(s, \omega)}|f(s, x(s, \omega), \omega)| d s+e^{-K(t, \omega)} \int_{0}^{t} e^{K(s, \omega)}|f(s, y(s, \omega), \omega)| d s \leq \\
& \leq 2 w(t)
\end{aligned}
$$

for all $t \in \mathbb{R}_{+}$. Since $\lim _{t \rightarrow \infty} w(t)=0$, there is a real number $T>0$ such that $w(t)<\frac{\varepsilon}{2}$ for all $t \geq T$. Therefore, $|x(t, \omega)-y(t, \omega)| \leq \varepsilon$ for all $t \geq T$ and for all $\omega \in \Omega$. Hence all random solutions of the RDE (1.1) are uniformly globally attractive on $\mathbb{R}_{+}$. 
Finally, we prove that random solutions are asymptotically attractive to the line $y=0$ on $\Omega \times \mathbb{R}_{+}$. Let $x: \Omega \rightarrow C\left(\mathbb{R}_{+}, \mathbb{R}\right)$ be a random solution of the RDE (1.1) on $\mathbb{R}_{+}$. Then, for each $\omega \in \Omega$,

$$
\begin{aligned}
|x(t, \omega)| & \leq|q(\omega)| e^{-K(t, \omega)}+e^{-K(t, \omega)} \int_{0}^{t} e^{K(s, \omega)}|f(s, x(s, \omega), \omega)| d s \leq \\
& \leq|q(\omega)| e^{-K(t, \omega)}+e^{-K(t, \omega)} \int_{0}^{t} e^{K(s, \omega)} h(s) d s \leq \\
& \leq c_{1} e^{-K(t, \omega)}+w(t)
\end{aligned}
$$

for all $\omega \in \Omega$. Taking the limit superior in the above inequality as $t$ tends to $\infty$ yields

$$
\limsup _{t \rightarrow \infty}|x(t, \omega)| \leq c_{1} \limsup _{t \rightarrow \infty} e^{-K(t, \omega)}+\limsup _{t \rightarrow \infty} w(t, \omega)=0
$$

and so, $\lim _{t \rightarrow \infty}|x(t, \omega)|=0$ for all $\omega \in \Omega$. Therefore, for each $\varepsilon>0$ there exists a real number $T>0$ such that $|x(t, \omega)|<\varepsilon$ for all $t \geq T$ and $\omega \in \Omega$. Hence, all random solutions of the RDE (1.1) are uniformly globally asymptotically attractive to the zero random solution on $\mathbb{R}_{+}$.

\section{PERTURBED RANDOM DIFFERENTIAL EQUATIONS}

Given a measurable space $(\Omega, \mathcal{A})$ and a measurable function $x: \Omega \rightarrow C\left(\mathbb{R}_{+}, \mathbb{R}\right)$, we consider the initial value problem of nonlinear first order ordinary perturbed random differential equations (in short PRDE)

$$
\left\{\begin{aligned}
\frac{d}{d t}[x(t, \omega)-g(t, x(t, \omega), \omega)] & +k(t, \omega)[x(t, \omega)-g(t, x(t, \omega), \omega)]= \\
& =f(t, x(t, \omega), \omega) \text { a.e. } t \in \mathbb{R}_{+}, \\
x(0, \omega) & =q(\omega)
\end{aligned}\right.
$$

for all $\omega \in \Omega$, where $k: \mathbb{R}_{+} \times \Omega \rightarrow \mathbb{R} \backslash\{0\}, q: \Omega \rightarrow \mathbb{R}$ and $f, g: \mathbb{R}_{+} \times \mathbb{R} \times \Omega \rightarrow \mathbb{R}$.

By a random solution of the $\operatorname{RDE}(5.1)$ we mean a measurable function $x: \Omega \rightarrow$ $A C\left(\mathbb{R}_{+}, \mathbb{R}\right)$ that satisfies the equations in $(5.1)$, where, as before, $A C\left(\mathbb{R}_{+}, \mathbb{R}\right)$ is the space of absolutely continuous real-valued functions defined on $\mathbb{R}_{+}$.

The differential equation (5.1) is a random or indeterministic version of the deterministic differential equation

$$
\left\{\begin{array}{c}
\frac{d}{d t}[x(t)-g(t, x(t))]+k(t)[x(t)-g(t, x(t))]=f(t, x(t)) \text { a.e. } t \in \mathbb{R}_{+}, \\
x(0)=q
\end{array}\right.
$$

where $k: \mathbb{R}_{+} \rightarrow \mathbb{R}$, and $f, g: \mathbb{R}_{+} \times \mathbb{R} \rightarrow \mathbb{R}$. 
The differential equation (5.2) with $k=1$ has been studied in Burton and Furumochi [2] for stability of solutions under some suitable growth condition via a fixed point theoretic technique of Krasnoselskii [9]. In this paper, we obtain the attractivity results for the PRDE (5.1) via a random version of a hybrid fixed point theorem of Dhage [3]. In what follows, we obtain the random version of the following indeterministic fixed point theorem of Dhage [3].

Theorem 5.1. Let $X$ be a closed, convex and bounded subset of a Banach space $E$ and let $A: E \rightarrow E$ and $B: X \rightarrow E$ be two operators such that:

(a) $A$ is a nonlinear contraction,

(b) $B$ is completely continuous, and

(c) $A x+B y=x, \forall y \in X \Longrightarrow x \in X$.

Then the operator equation $A x+B x=x$ has a solution.

Definition 5.2. A random operator $Q: \Omega \times E \rightarrow E$ is called $\mathcal{D}$-Lipschitz if there exists a continuous nondecreasing function $\phi_{\omega}: \mathbb{R}^{+} \rightarrow \mathbb{R}^{+}$such that for each $\omega \in \Omega$,

$$
\|Q(\omega) x-Q(\omega) y\| \leq \phi_{\omega}(\|x-y\|)
$$

for all $x, y \in E$, where $\phi_{\omega}(0)=\phi(\omega, 0)=0$ for all $\omega \in \Omega$. Sometimes we call the function $\phi$ to be a $\mathcal{D}$-function of $Q(\omega)$ on $E$. In the special case, when $\phi_{\omega}(r)=$ $\phi(\omega, r)=k(\omega) r$ for some measurable function $k: \Omega \rightarrow(0, \infty), Q(\omega)$ is called a $k$-Lipschitz and if $k(\omega)<1$ for all $\omega \in \Omega$, then $Q(\omega)$ is called a $k$-contraction random operator on $E$. Further, if $\phi_{\omega}(r)=\phi(\omega, r)<r$ for $r>0$ and for all $\omega \in \Omega$, then $Q(\omega)$ is called a nonlinear $\mathcal{D}$-contraction random operator on $E$.

Theorem 5.3. Let $X$ be a closed, convex and bounded subset of a separable Banach space $E$ and let $A: \Omega \times E \rightarrow E$ and $B: \Omega \times X \rightarrow E$ be two operators such that for each $\omega \in \Omega$ :

(a) $A(\omega)$ is a nonlinear contraction,

(b) $B(\omega)$ is completely continuous, and

(c) $A(\omega) x+B(\omega) y=x, \forall y \in X \Longrightarrow x \in X$.

Then the operator equation $A(\omega) x+B(\omega) x=x$ has a random solution.

Proof. Let $\omega \in \Omega$ be fixed and define a multi-valued map $F: \Omega \rightarrow \mathcal{P}_{p}(E)$ by

$$
F(\omega)=\{x \in X \mid A(\omega) x+B(\omega) x=x\} .
$$

Clearly by Theorem 5.1, $F(\omega)$ is non-empty for each $\omega \in \Omega$. To finish, it is enough to prove that $F$ is measurable on $\Omega$. Let $C$ be a closed subset of $X$. Denote

$$
L(C)=\bigcap_{n=1}^{\infty} \bigcup_{x_{i} \in C_{n}}\left\{\omega \in \Omega \mid\left\|x_{i}-\left(A(\omega) x_{i}+B(\omega) x_{i}\right)\right\|<2 / n\right\},
$$

where

$$
C_{n}=\{x \in X \mid d(x, C)<1 / n\}
$$


and

$$
d(x, C)=\inf \{d(x, c) \mid c \in C\} .
$$

Obviously $L(C) \in \mathcal{A}$. We shall prove that $F^{-1}(C)=L(C)$. It is easy to prove that $F^{-1}(C) \subset L(C)$. Now proceeding with the arguments similar to that given in the proof of Theorem 2.1 of Itoh [8], it is proved that $L(C) \subset F^{-1}(C)$. As a result $F^{-1}(C)=L(C)$. Hence $F$ is measurable on $\Omega$. Next we show that $F(\omega)$ is closed for each $\omega \in \Omega$. Let $\left\{x_{n}\right\}$ be a sequence of points in $F(\omega)$ converging to a point $x$. Since every nonlinear contraction random operator is continuous, we have

$$
A(\omega) x+A(\omega) x=\lim _{n \rightarrow \infty} A(\omega) x_{n}+\lim _{n \rightarrow \infty} B(\omega) x_{n}=\lim _{n \rightarrow \infty} x_{n}=x .
$$

As a result, $x \in F(\omega)$ which thereby implies that $F(\omega)$ is closed for each $\omega \in \Omega$. Hence, the mapping $F$ has closed values on $\Omega$ into $X$. Now an application of the measurable selector theorem of Kurtowskii and Ryll-Nardzewskii (see Itoh [8] and the references given therein) yields that $F$ has a measurable selector, that is, there is a measurable mapping $\xi: \Omega \rightarrow X$ such that $\xi(\omega) \in F(\omega)$ for each $\omega \in \Omega$. Further by the definition of $F(\omega)$ this implies that

$$
\xi(\omega)=A(\omega) \xi(\omega)+B(\omega) \xi(\omega)), \quad \forall \omega \in \Omega .
$$

This completes the proof.

Let $X=B C\left(\mathbb{R}_{+}, \mathbb{R}\right)$ and let $\mathcal{M}(\Omega, X)$ be the class of measurable $X$-valued functions on $\Omega$. We define the norm $\|\cdot\|_{\infty}$ in $\mathcal{M}(\Omega, X)$ by

$$
\|\cdot\|_{\infty}=\operatorname{ess} \sup _{\omega \in \Omega}\|x(\omega)\| .
$$

Two norms $\|\cdot\|$ and $\|\cdot\|_{\infty}$ are said to be comparable if there exists a constant $c>0$ such that

$$
c\|\cdot\| \leq\|\cdot\|_{\infty} \leq\|\cdot\| .
$$

We need the following hypotheses in what follows.

$\left(\mathrm{H}_{3}\right)$ The function $g: \mathbb{R}_{+} \times \mathbb{R} \times \Omega \rightarrow \mathbb{R}$ is measurable and bounded. Moreover, we assume that

$$
G_{q}=\operatorname{ess}_{\sup _{\omega \in \Omega}}[g(0, q(\omega), \omega)]
$$

and

$$
G_{0}=\operatorname{ess}_{\sup } \sup _{\omega \in \Omega}\left[\sup _{t \in \mathbb{R}_{+}} g(t, 0, \omega)\right] .
$$

$\left(\mathrm{H}_{4}\right)$ The norms $\|\cdot\|$ and $\|\cdot\|_{\infty}$ are comparable and there exists a measurable function $\ell: \mathbb{R}_{+} \times \Omega \rightarrow \mathbb{R}$ and a positive constant $K$, such that for each $\omega \in \Omega$,

$$
|g(t, x, \omega)-g(t, y, \omega)| \leq \frac{\ell(t, \omega)|x-y|}{K+|x-y|}
$$

for all $t \in \mathbb{R}_{+}$and $x, y \in \mathbb{R}$. Moreover, we assume that

$$
\lim _{t \rightarrow \infty} \ell(t, \omega)=0 \text { for all } \omega \in \Omega,
$$


and

$$
\operatorname{ess} \sup _{\omega \in \Omega}\left[\sup _{t \in \mathbb{R}_{+}} \ell(t, \omega)\right]=L .
$$

Theorem 5.4. Assume that the hypotheses $\left(\mathrm{H}_{0}\right)$ through $\left(\mathrm{H}_{4}\right)$ hold. Further, if $L \leq K$, then the PRDE (5.1) admits a random solution and solutions are globally uniformly attractive on $\mathbb{R}_{+}$.

Proof. Now PRDE (5.1) is equivalent to the random equation

$$
\begin{aligned}
x(t, \omega)= & {[q(\omega)-g(0, q(\omega), \omega)] e^{-K(t, \omega)}+g(t, x(t, \omega), \omega)+} \\
& +e^{-K(t, \omega)} \int_{0}^{t} e^{K(s, \omega)} f(s, x(s, \omega), \omega) d s
\end{aligned}
$$

for all $t \in \mathbb{R}_{+}$and $\omega \in \Omega$.

Set $E=B C\left(\mathbb{R}_{+}, \mathbb{R}\right)$ and define a closed ball $\overline{\mathcal{B}}_{r}(0)$ centered at the origin of radius $r=\|q\|+G_{q}+G_{0}+L+W$. Define two operators $A: \Omega \times E \rightarrow E$ and $B: \Omega \times \overline{\mathcal{B}}_{r}(0) \rightarrow E$ by

$$
A(\omega) x(t)=g(t, x(t, \omega), \omega)
$$

and

$$
\begin{aligned}
B(\omega) x(t)= & {[q(\omega)-g(0, q(\omega), \omega)] e^{-K(t, \omega)}+} \\
& +e^{-K(t, \omega)} \int_{0}^{t} e^{K(s, \omega)} f(s, x(s, \omega), \omega) d s
\end{aligned}
$$

for all $t \in \mathbb{R}_{+}$and $\omega \in \Omega$.

From $\left(\mathrm{H}_{0}\right)$ it follows that $A(\omega)$ is a random operator on $E$ into itself. Similarly, it can be shown as in the proof of Theorem 4.3 that $B(\omega)$ is also a random operator on $\overline{\mathcal{B}}_{r}(0) E$. We will show that $A(\omega)$ and $B(\omega)$ satisfy all the conditions of Theorem 5.3 .

Firstly, we show that $A(\omega)$ is a nonlinear random contraction operator on $E$. Let $x, y \in E$. Then, by hypothesis $\left(\mathrm{H}_{4}\right)$, we obtain

$$
\begin{aligned}
|A(\omega) x(t)-A(\omega) y(t)| & =|g(t, x(t, \omega), \omega)-g(t, y(t, \omega), \omega)| \leq \\
& \leq \frac{\ell(t, \omega)|x(t, \omega)-y(t, \omega)|}{K+|x(t, \omega)-y(t, \omega)|} \leq \frac{L\|x-y\|}{K+\|x-y\|}
\end{aligned}
$$

for all $\omega \in \Omega$. Taking supremum over $t$,

$$
\|A(\omega) x-A(\omega) y\| \leq \frac{L\|x-y\|}{K+\|x-y\|}
$$

for all $\omega \in \Omega$. Since $\phi(r)=\frac{L r}{K+r}<r$ for $r>0$, one has $A(\omega)$ is a nonlinear contraction random operator on $E$ into itself. 
Next, it can proved as in the proof of Theorem 4.3 that $B(\omega)$ is a completely continuous random operator on $\overline{\mathcal{B}}_{r}(0)$ into $E$. Finally, assume that $x=A(\omega) x+B(\omega) y$ for all $y \in \overline{\mathcal{B}}_{r}(0)$. Then,

$$
\begin{aligned}
|x(t, \omega)| \leq & |A(\omega) x(t)|+|B(\omega) y(t)| \leq \\
\leq & |g(t, x(t, \omega), \omega)|+|q(\omega)-g(0, q(\omega), \omega)| e^{-K(t, \omega)}+ \\
& +e^{-K(t, \omega)} \int_{0}^{t} e^{K(s, \omega)}|f(s, x(s, \omega), \omega)| d s \leq \\
\leq & |g(t, x(t, \omega), \omega)-g(t, 0, \omega)|+|g(t, 0, \omega)|+ \\
& +|q(\omega)|+|g(0, q(\omega), \omega)|+e^{-K(t, \omega)} \int_{0}^{t} e^{K(s, \omega)} h(s) d s \leq \\
\leq & \frac{L|x(t, \omega)|}{K+|x(t, \omega)|}+G_{0}+\|q\|+G_{q}+W \leq \\
\leq & L+G_{0}+\|q\|+G_{q}+W
\end{aligned}
$$

for all $t \in \mathbb{R}_{+}$and $\omega \in \Omega$. This shows that $x(\omega) \in \overline{\mathcal{B}}_{r}(0)$ for all $\omega \in \Omega$.

Thus, all the conditions of Theorem 5.3 are satisfied. Hence an application of it yields that the random operator equation $A(\omega) x+B(\omega) x=x$ has a random solution. As a result, the PRDE (5.1) has a random solution defined on $\mathbb{R}_{+}$.

Let $x, y: \Omega \rightarrow \overline{\mathcal{B}}_{r}(0)$ be any two random solutions to the $\operatorname{RDE}(5.1)$ on $\mathbb{R}_{+}$. Then, for each $\omega \in \Omega$,

$$
\begin{aligned}
|x(t, \omega)-y(t, \omega)| \leq & |g(t, x(t, \omega), \omega)-g(t, x(t, \omega), \omega)|+ \\
& +\mid e^{-K(t, \omega)} \int_{0}^{t} e^{K(s, \omega)} f(s, x(s, \omega), \omega) d s- \\
& -e^{-K(t, \omega)} \int_{0}^{t} e^{K(s, \omega)} f(s, y(s, \omega), \omega) d s \mid \leq \\
\leq & \frac{\ell(t, \omega)|x(t, \omega)-y(t, \omega)|}{K+|x(t, \omega)-y(t, \omega)|}+ \\
& +e^{-K(t, \omega)} \int_{0}^{t} e^{K(s, \omega)}|f(s, x(s, \omega), \omega)| d s+ \\
& +e^{-K(t, \omega)} \int_{0}^{t} e^{K(s, \omega)}|f(s, y(s, \omega), \omega)| d s \leq \\
\leq & \ell(t, \omega)+2 w(t, \omega)
\end{aligned}
$$

for all $t \in \mathbb{R}_{+}$. 
Since $\lim _{t \rightarrow \infty} \ell(t, \omega)=0$ and $\lim _{t \rightarrow \infty} w(t, \omega)=0$ for each $\omega \in \Omega$, there are real numbers $T_{1}>0$ and $T_{2}>0$ such that $\ell(t, \omega)<\frac{\varepsilon}{2}$ for all $t \geq T_{1}$ and $w(t, \omega)<\frac{\varepsilon}{4}$ for all $t \geq T_{2}$ and for all $\omega \in \Omega$. Choose $T=\max \left\{T_{1}, T_{2}\right\}$. Then, $|x(t, \omega)-y(t, \omega)| \leq \varepsilon$ for all $t \geq T$ and for all $\omega \in \Omega$. Hence all random solutions of the PRDE (5.1) are uniformly globally attractive on $\mathbb{R}_{+}$.

The conclusion of Theorem 5.4 also remains true if we replace the hypothesis $\left(\mathrm{H}_{4}\right)$ with the following general one.

$\left(\mathrm{H}_{5}\right)$ The norms $\|\cdot\|$ and $\|\cdot\|_{\infty}$ are comparable and there exists a measurable function $\ell: \mathbb{R}_{+} \times \Omega \rightarrow \mathbb{R}$ and a continuous, nondecreasing function $\phi: \mathbb{R}_{+} \rightarrow \mathbb{R}_{+}$such that for each $\omega \in \Omega$,

$$
|g(t, x, \omega)-g(t, y, \omega)| \leq \ell(t, \omega) \phi(|x-y|)
$$

for all $t \in \mathbb{R}_{+}$and $x, y \in \mathbb{R}$ and

$$
\lim _{t \rightarrow \infty} \ell(t, \omega)=0 \text { and } L \phi(r)<r
$$

for all $r>0$, where ess $\sup _{\omega \in \Omega}\left[\sup _{t \in \mathbb{R}_{+}} \ell(t, \omega)\right]=L$.

\section{EXAMPLES}

Example 6.1. Let $\Omega=(-\infty, 0)$. Given a function $x: \mathbb{R}_{+} \times \Omega \rightarrow C\left(\mathbb{R}_{+}, \mathbb{R}\right)$, consider the RDE

$$
\left\{\begin{array}{c}
x^{\prime}(t, \omega)+x(t, \omega)=\frac{e^{-t} \sin \omega t x(t, \omega)}{1+|x(t, \omega)|} \\
x(0, \omega)=1
\end{array}\right.
$$

for all $t \in \mathbb{R}_{+}$and $\omega \in \Omega$.

Here,

$$
q(\omega)=1, k(t, \omega)=1 \text { for all } \omega \in \Omega,
$$

and

$$
f(t, x, \omega)=\frac{e^{-t} \sin \omega t x}{1+|x|}
$$

for $t \in \mathbb{R}_{+}, x \in \mathbb{R}$ and $\omega \in(-\infty, 0)$. Clearly, the function $f$ is random $L^{1}$-Carathéodory with growth function

$$
h(t)=e^{-t} \geq\left|\frac{e^{-t} \sin \omega t x}{1+|x|}\right|=|f(t, x, \omega)| .
$$

Again, we have

$$
\lim _{t \rightarrow \infty} e^{-t} \int_{0}^{t} e^{-s} e^{s} d s=\lim _{t \rightarrow \infty} e^{-t} \int_{0}^{t} d s=\lim _{t \rightarrow \infty} \frac{t}{e^{t}}=0 .
$$


Thus, both the hypotheses $\left(\mathrm{H}_{0}\right)$ and $\left(\mathrm{H}_{2}\right)$ of Theorem 4.3 are satisfied and hence the RDE (6.1) has a random solution and all random solutions are uniformly globally asymptotically attractive to the zero random solution defined on $\mathbb{R}_{+}$.

Example 6.2. Let $\Omega=(-\infty, 0)$. Given a function $x: \mathbb{R}_{+} \times \Omega \rightarrow C\left(\mathbb{R}_{+}, \mathbb{R}\right)$, consider the RDE

$$
\left\{\begin{array}{c}
\frac{d}{d t}\left[x(t, \omega)-\frac{1}{t+1} \tan ^{-1} x(t, \omega)\right] \\
+\left[x(t, \omega)-\frac{1}{t+1} \tan ^{-1} x(t, \omega)\right]=\frac{e^{-t} \sin \omega t x(t, \omega)}{1+|x(t, \omega)|} \\
x(0, \omega)=1
\end{array}\right.
$$

for all $t \in \mathbb{R}_{+}$and $\omega \in \Omega$.

Here,

$$
q(\omega)=1, k(t, \omega)=1, g(t, x, \omega)=\frac{1}{t+1} \tan ^{-1} x(t, \omega) \text { and } f(t, x, \omega)=\frac{e^{-t} \sin \omega t x}{1+|x|}
$$

for $t \in \mathbb{R}_{+}, x \in \mathbb{R}$ and $\omega \in(-\infty, 0)$. Clearly, the functions $q$ and $f$ satisfy hypotheses $\left(\mathrm{H}_{0}\right)$ and $\left(\mathrm{H}_{2}\right)$ with growth function $h(t)=e^{-t}$. Again, hypotheses $\left(\mathrm{H}_{3}\right)$ and $\left(\mathrm{H}_{4}\right)$ hold with $\ell(t)=\frac{1}{t+1}$. To see this, let $x, y \in \mathbb{R}$. Then,

$$
|g(t, x, \omega)-g(t, y, \omega)| \leq \frac{1}{t+1}\left|\tan ^{-1} x-\tan ^{-1} y\right| \leq \frac{1}{t+1} \phi(|x-y|)
$$

for all $t \in \mathbb{R}_{+}$and $\omega \in \Omega$, where the function $\phi: \mathbb{R}_{+} \rightarrow \mathbb{R}_{+}$is defined by

$$
\phi(|x-y|)=\frac{|x-y|}{1+\xi^{2}}, x<\xi<y
$$

Now,

$$
\lim _{t \rightarrow \infty} \ell(t)=0 \quad \text { and } \quad \sup _{t \geq 0} \frac{1}{t+1}=1=K,
$$

and so, the hypothesis $\left(\mathrm{H}_{4}\right)$ holds. Moreover,

$$
\lim _{t \rightarrow \infty} e^{-t} \int_{0}^{t} e^{-s} e^{s} d s=\lim _{t \rightarrow \infty} e^{-t} \int_{0}^{t} d s=\lim _{t \rightarrow \infty} \frac{t}{e^{t}}=0 .
$$

Thus, all the hypotheses of Theorem 5.4 are satisfied and hence the RDE (6.1) has a random solution and all random solutions are uniformly globally attractive solutions defined on $\mathbb{R}_{+}$. 


\section{CONCLUSION}

In this paper, we have been able to extend and generalize some known attractivity or stability results of deterministic nonlinear differential equations obtained in Burton and Furumochi [2] to indeterministic case of random differential equations on unbounded intervals of real line. Further, the ideas of this paper may be used to prove the similar results for a variety of nonlinear random differential and integral equations with appropriate modifications. These and other similar problems form the further scope for research work in the theory of random differential equations. We conclude this paper by mentioning that a few results along these lines will be reported elsewhere.

\section{REFERENCES}

[1] A.T. Bharucha-Reid, Random Integral Equations, Academic Press, New York, 1972.

[2] T.A. Burton, T. Furumochi, A note on stability by Schauder's theorem, Funkcial. Ekvac. 44 (2001), 73-82.

[3] B.C. Dhage, A nonlinear alternative with applications to nonlinear perturbed differential equations, Nonlinear Stud. 13 (2006) 4, 343-354.

[4] B.C. Dhage, Some algebraic and topological random fixed point theorems with applications to nonlinear random integral equations, Tamkang J. Math. 35 (2004), 321-345.

[5] B.C. Dhage, Local asymptotic attractivity for nonlinear quadratic functional integral equations, Nonlinear Anal. 70 (2009) 5, 1912-1922.

[6] B.C. Dhage, On global existence and attractivity results for nonlinear random integral equations, Panamer. Math. J. 19 (2009) 1, 97-111.

[7] X. Hu, J. Yan, The global attractivity and asymptotic stability of solution of a nonlinear integral equation, J. Math. Anal. Appl. 321 (2006), 147-156.

[8] S. Itoh, Random fixed point theorems with applications to random differential equations in Banach spaces, J. Math. Anal. Appl. 67 (1979), 261-273.

[9] M.A. Krasnoselskii, Topological Methods in the Theory of Nonlinear Integral Equations, Macmillan, New York, 1964 [translated from the Russian].

Bapurao C. Dhage

bcdhage@yahoo.co.in

Kasubai, Gurukul Colony

Ahmedpur-413 515, Dist: Latur, Maharashtra, India

Sotiris K. Ntouyas

sntouyas@uoi.gr 
University of Ioannina

Department of Mathematics

45110 Ioannina, Greece

Received: April 2, 2010.

Revised: April 17, 2010.

Accepted: April 17, 2010. 